\title{
AN INTERACTING MULTIPLE MODEL ALGORITHM FOR STOCHASTIC SYSTEMS CONTROL
}

\author{
Ludmila MIHAYLOVA and Emil SEMERDJIEV
}

\section{Introduction}

During the last years the multiple-model (MM) approach has become very popular and widely applied for estimation ${ }^{2,5,7,9}$ and control ${ }^{1,3,4,6-8,10-12}$ of stochastic systems under different kinds of uncertainty - unknown model structure or parameters. In the engineering applications different multiple model algorithms for system control have been proposed. ${ }^{3,10,11}$ The greatest number of them are of Bayesian nature. ${ }^{1,3,4,7,8,10,12}$ Their common feature is the presence of a bank of estimators providing separate state estimates required for the overall control synthesis. But, the Interacting Multiple Model estimator - the most cost-effective scheme for solving various problems for state and parameter estimation, ${ }^{2,5,13}$ has not yet been used to solve problems for systems control.

In the present paper an IMM algorithm is designed for control of stochastic systems in the presence of parametric model uncertainty. The overall system control is formed as a probabilistically weighted sum of the control processes provided by separate regulators. Regulators are synthesized for a set of respective models covering the uncertainty domain. These regulators optimize a quadratic cost function.

The separate state estimates, generated by a modified IMM estimation algorithm and based on the same models, represent the respective regulators' inputs. The model probabilities are the weighting coefficients for the separate control processes, each computed as a full-state feedback. The algorithm performance is evaluated through Monte Carlo simulation experiments and compared to other MM algorithms for control. 


\section{IMM algorithm for systems control}

The system is described by the model:

$$
\begin{aligned}
& x(k+1)=F(k, m(k+1)) x(k)+G_{u}(k, m(k+1)) u(k)+ \\
& +G_{v}(k, m(k+1)) v(k, m(k+1)), \\
& z(k)=H(k) x(k)+w(k),
\end{aligned}
$$

where $x \in \mathfrak{R}^{n_{x}}$ is the system state vector, $z \in \mathfrak{R}^{n_{z}}$ is the measurement vector; $u \in \mathfrak{R}^{n_{u}}$ - the control input vector; $v \in \mathfrak{R}^{n_{v}}$ and $w \in \mathfrak{R}^{n_{z}}$ are mutually uncorrelated, white, zero mean Gaussian noises with covariances $D_{v}$ and $D_{w}$, respectively. The parameter $m_{k}$ presents the current system mode. The structure of the model (1) is supposed known, but its parameters belong to an uncertainty domain and are assumed to depend on different system modes.

The problem consists in synthesizing a control sequence $\left\{u_{k}\right\}$, so that the quadratic cost function

$$
J=M\left\{\sum_{k=0}^{\infty}\left[x^{T}(k) Q x(k)+u^{T}(k) R u(k)\right]\right\},
$$

is minimized, where $Q$ and $R$ are appropriately chosen weighting matrices ( $Q$ positive semi-definite, $R$ - positive definite) and $M\{$.$\} is the mathematical$ expectation operator.

Because the accurate system model is unknown, the system is described by a number of models from the preliminary determined uncertainty domain. The event that the $i$ th model $m_{i}$ is actual at time $k$ is denoted as

$$
m_{i}(k)=\left\{m(k)=m_{i}\right\} \text {. }
$$

It is assumed that the system model sequence is a Markov chain with transition probabilities

$$
P\left\{m_{j}(k+1) / m_{i}(k)\right\}=P r_{i j}(k)
$$

and

$$
\sum_{j=1}^{q} \operatorname{Pr}_{i j}(k)=1, \quad i=1,2, \ldots, q
$$


The main functional components of the IMM algorithm for control are:

separate estimators - Kalman filters (KF), running in parallel and providing the input signals (the partial state estimates) for the regulators;

separate regulators, generating the single-model-based control processes.

The control process of the system is computed as a state feedback:

$$
u(k)=-\sum_{i=1}^{q} \mu_{i}(k) K_{r, i}\left(k ; m_{i}\right) \hat{x}_{i}(k),
$$

where $\mu_{i}$ are the IMM mode probabilities, $K_{r, i}$ - the matrices of the regulators working in parallel, $\hat{x}_{i}$ are partial state estimates generated by $q$ Kalman filters. The partial regulators are linear quadratic Gaussian (LQG). The matrices $K_{r, i}$ are computed through minimization of the cost function $J$ for each system model $m_{i}\left(F_{i}, G_{u, i}, G_{\mathrm{v}, \mathrm{i}}, H_{i}, D_{v, i}, D_{w, i}\right), \quad i=1,2, \cdots, q$ with matrices chosen from the uncertainty domain. The regulators' gains are generated by solving the Riccati difference equations.

The overall state estimate $\hat{x}_{k}$, generated by the IMM estimator, has the form:

$$
\hat{x}(k)=\sum_{i=1}^{q} \mu_{i}(k) \hat{x}_{i}(k) \text {. }
$$

This standard IMM algorithm step for separate estimates combination is excluded here.

Each IMM partial state estimate $\hat{x}_{i, k}(i=1,2, \cdots, q)$ is computed by a respective Kalman filter:

$$
\begin{aligned}
& \hat{x}_{i}(k+1 / k)=F_{i}(k) \hat{x}_{i}(k / k)+G_{u, i}(k) u(k), \\
& \hat{x}_{i}(k+1 / k+1)=\hat{x}_{i}(k+1 / k)+K_{f, i}(k+1) v_{i}(k+1), \\
& v_{i}(k+1)=z(k+1)-H_{i}(k+1) \hat{x}_{i}(k+1 / k), \\
& P_{i}(k+1 / k)=F_{i}(k) P_{i}(k / k) F_{i}^{T}(k)+G_{v, i}(k) D_{v, i}(k) G_{v, i}^{T}(k), \\
& S_{i}(k+1)=H_{i}(k+1) P_{i}(k+1 / k)_{i} H_{i}^{T}(k+1)+D_{w, i}(k+1), \\
& K_{i}(k+1)=P_{i}(k+1 / k) H_{i}^{T}(k+1) S_{i}^{-1}(k+1), \\
& P_{i}(k+1 / k+1)=P_{i}(k+1 / k)-K_{i}(k+1) S_{i}(k+1) K_{i}^{T}(k+1),
\end{aligned}
$$

where $\hat{x}_{i}(k / k)$ and $\hat{x}_{i}(k / k-1)$ are the filtered and predicted estimates of $x(k) ; v_{i}, S_{i}$ are the innovation process and its covariance matrix; $K_{f, i}$ - the filter gain; $P_{i}$ - the error covariance matrix. 


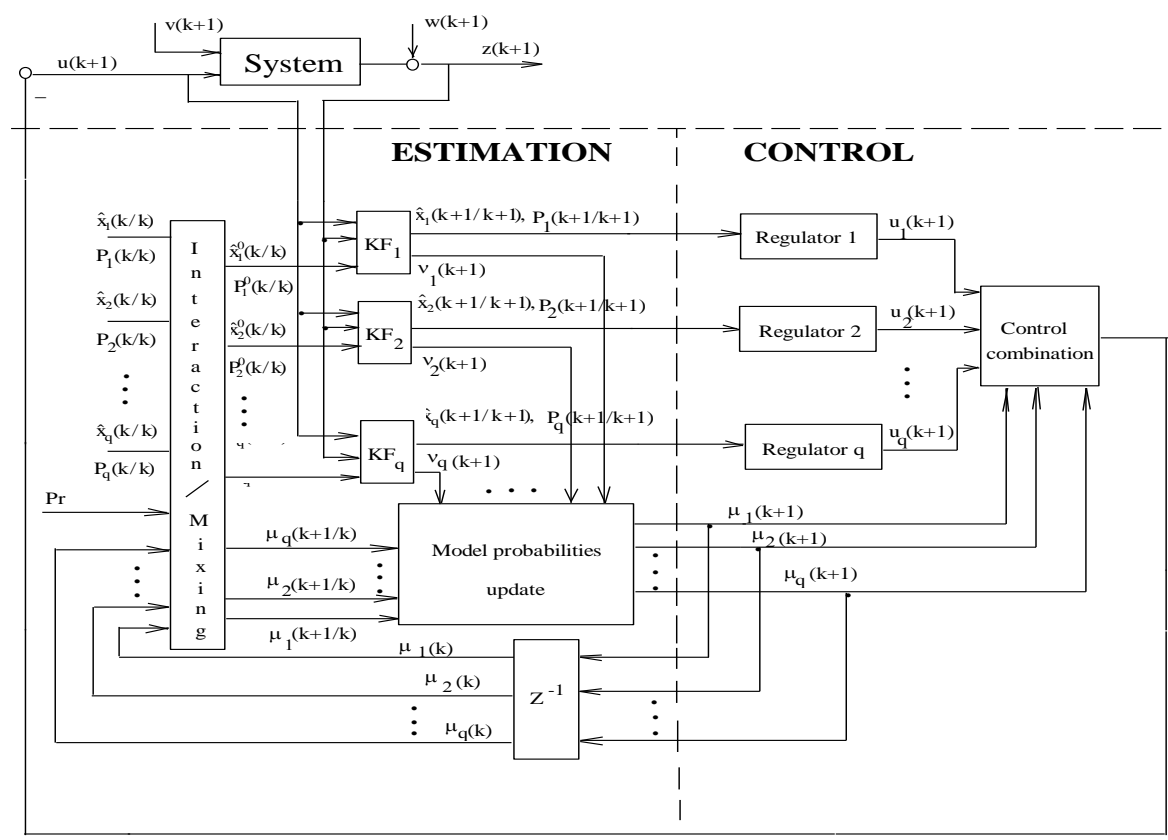

Figure 1: Scheme of the IMM algorithm for control

Figure 1 shows the scheme of the proposed IMM algorithm for control. Similarly to the IMM estimator, ${ }^{2,5,13}$ the IMM algorithm for control comprises the four major steps:

- interacting or mixing of the state estimates and their covariances, respectively;

- model-conditional filtering, performed in parallel for each mode through a respective Kalman filter - eqs. (6)-(12);

- model probability update, based on the model conditional likelihood functions;

- control combination, according to (4), which yields the overall control process as the probabilistically weighted sum of the control processes, provided by the regulators.

\section{Comparison with other MM control algorithms}

The main difference between the proposed IMM algorithm for control with respect to other MM control algorithms ${ }^{1,3,4,6,7,8,11,12}$ relies on the nature of the Interacting Multiple Model approach. ${ }^{2,5,13}$ The main feature is the dynamic interaction between the single-model filters, obtained by mixing the estimates of all filters at the previous step and using the mixed estimate as initial estimate for the filters in the next step. A 
very important is the assumption that the transition between the different controlled regimes can be described as a Markov process and it is reflected in the transition probability matrix. The presented control algorithm is compared in the next section with the Multiple Model Adaptive Controller (MMAC), proposed in ${ }^{8,12}$. The two algorithms are characterized by the same multiple model structure, they are of Bayesian type, but the mechanism for model probabilities computation is different. A comparison of the IMM estimator for detection and diagnosis of sensor and actuator failures with the MM adaptive estimator (MMAE) ${ }^{8}$ is performed in ${ }^{14}$.

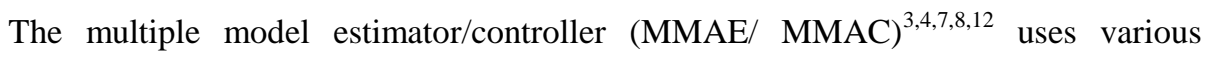
heuristic techniques, such as Kalman filtering retuning and bounded conditional mode probabilities. The effect termed "lockout" of these probabilities can be observed in the MMAC algorithm. It expresses itself in probabilities going to zero, that is why an additional lower bound of these probabilities is predetermined.

The decision thresholds for moving of the bank of filters/ controllers are also determined empirically. These techniques enhance the performance of the MMAE/MMAC in an empirical fashion. In contrast to them the IMM algorithm for control is working without additional tuning procedures.

\section{Performance evaluation}

Results illustrating the efficiency of the proposed IMM algorithm for stochastic systems control are given. Its performance is evaluated by Monte Carlo simulation experiments for 100 runs and compared to the MMAC algorithm performance, presented in ${ }^{8,12}$. The MMAC algorithm is implemented in the simulation experiments with an artificial lower bound for the mode probabilities $\mu_{\text {min }}=0.001$, as imposed in ${ }^{8}$. In the example the overall control for both algorithms is synthesized based on the same steady-state constant gain regulators.

Example. The proposed IMM algorithm for control is applied to a paper machine ${ }^{14}$ producing a super-thin condenser paper. The state space model of its headbox section ${ }^{14}$ has the form:

$$
x(k+1)=F x(k)+G_{u} u(k)+G_{v} v(k),
$$

where

$$
F=\left(\begin{array}{ccc}
0.8667 & 0 & 0 \\
0 & 0.8667 & 0 \\
0.1069 & -0.0503 & 0.9099
\end{array}\right), G_{u}=\left(\begin{array}{cc}
-0.0344 & 0 \\
0 & -0.6877 \\
0 & 0
\end{array}\right),
$$

$G_{v}=I_{3}$ and $I$ is the identity matrix. The measurement matrix and the noise covariances are: 


$$
H=\left(\begin{array}{lll}
1 & 0 & 0 \\
0 & 0 & 1
\end{array}\right), D_{v}=\operatorname{diag}(0.16,0.16,1), D_{w}=\operatorname{diag}(0.09,0.16)
$$

It is supposed that the system matrix $F$ is inaccurately known. The model uncertainty domain here is approximated by four models:

$$
\begin{aligned}
& F_{1}=\left(\begin{array}{ccc}
0.1000 & 0 & 0 \\
0 & 0.1000 & 0 \\
0.1069 & -0.0503 & 0.9099
\end{array}\right), F_{2}=\left(\begin{array}{ccc}
0.3000 & 0 & 0 \\
0 & 0.3000 & 0 \\
0.1069 & -0.0503 & 0.9099
\end{array}\right) ; \\
& F_{3}=\left(\begin{array}{ccc}
0.5000 & 0 & 0 \\
0 & 0.5000 & 0 \\
0.1069 & -0.0503 & 0.9099
\end{array}\right), F_{4}=\left(\begin{array}{ccc}
0.8000 & 0 & 0 \\
0 & 0.8000 & 0 \\
0.1069 & -0.0503 & 0.9099
\end{array}\right) .
\end{aligned}
$$

The other model matrices coincide with the true model matrices. The fourth model is the closest to the true one. The matrices of the quadratic cost function are chosen to provide rapid transient processes of the closed-loop system: $Q=100 I_{3}$, $R=0.01 I_{2}$.

The IMM transition probability matrix and the initial mode probability vector are chosen :

$$
\operatorname{Pr}=\left(\begin{array}{llll}
0.997 & 0.001 & 0.001 & 0.001 \\
0.001 & 0.997 & 0.001 & 0.001 \\
0.001 & 0.001 & 0.997 & 0.001 \\
0.001 & 0.001 & 0.001 & 0.997
\end{array}\right), \mu(0)=\left(\begin{array}{l}
1 / 4 \\
1 / 4 \\
1 / 4 \\
1 / 4
\end{array}\right)
$$

The following measures of performance are used:

- the recursively computed cost function $J$ (Fig. 2), as instead of the true state $x$ in (3) its overall estimate $\hat{x}$ is replaced;

- $\quad$ the averaged algorithm mode probabilities (shown in Figs. 3 and 4).

It is denoted below: "1" - the IMM algorithm for control and "2" - the MMAC algorithm. ${ }^{8}$ 


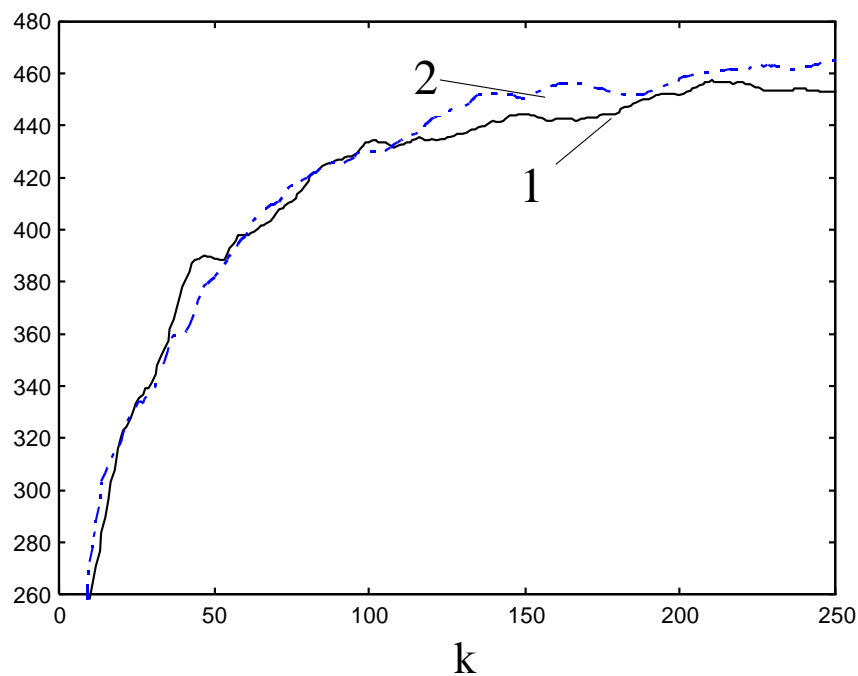

Figure 2: Cost function $J$

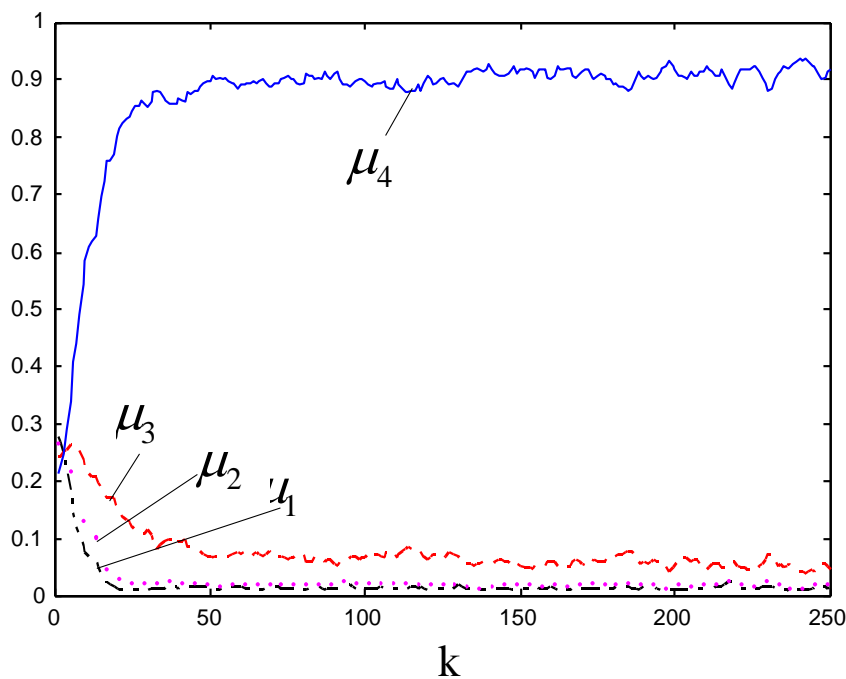

Figure 3: Average IMM mode probabilities 


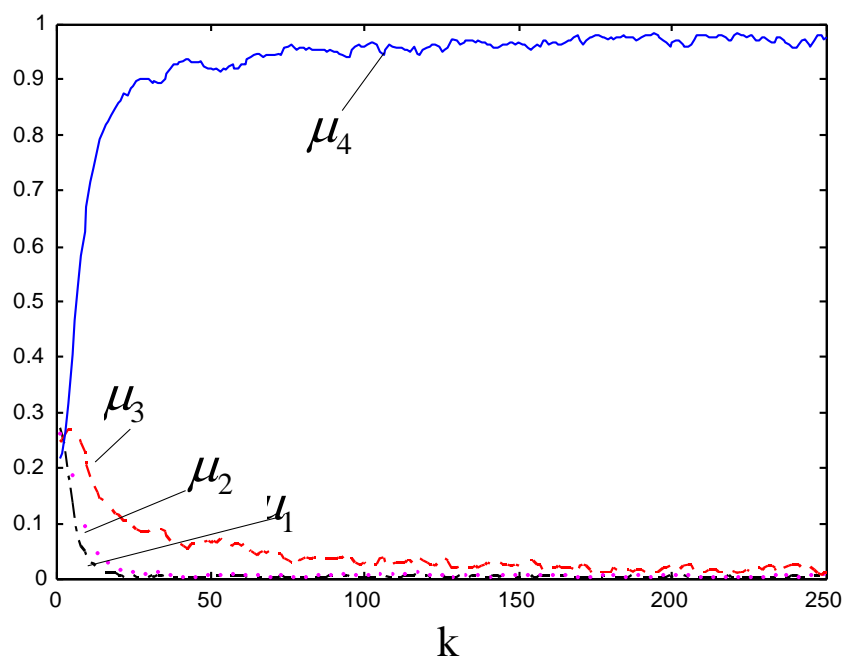

Figure 4: Average MMAC mode probabilities

In the considered here stationary example both algorithms show nearly equal performance: they quickly recognize the fourth model as the closest to the true one (its probability is the greatest).

The test scenario has been artificially complicated to evaluate the algorithm performance in the nonstationary case. In the next scenario abrupt changes arise in elements of the matrix $F$ :

$$
F=\left\{\begin{array}{c}
\text { true matrix } F \text {, for } k<50 \\
F_{4}, \text { for } 50 \leq k<70 \\
F_{3}, \text { for } 70 \leq k<150 \\
F_{1}, \quad \text { for } k \geq 150
\end{array} .\right.
$$

The computed cost function $J$ and the mode probabilities are presented in Figs. 5-7. 


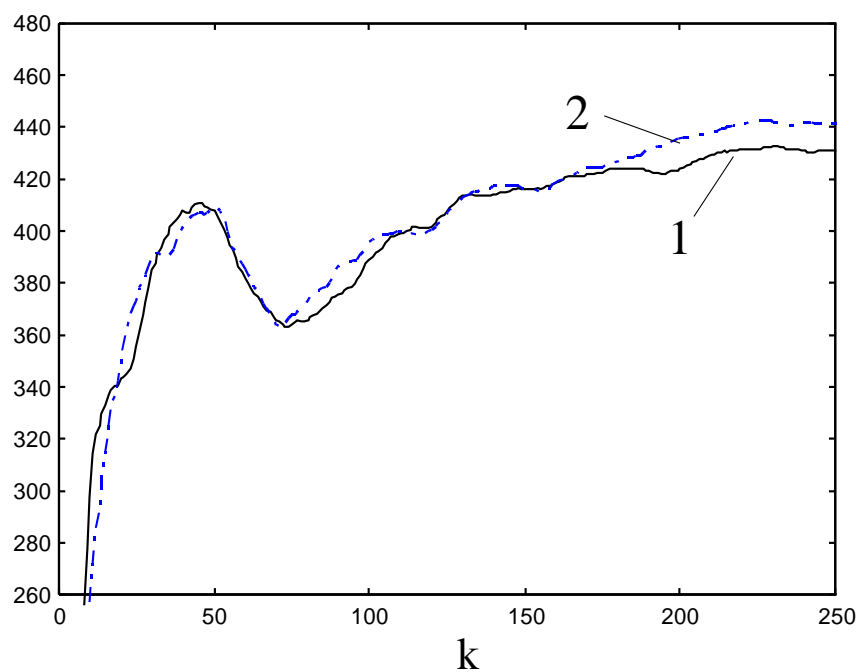

Figure 5: Cost function $J$

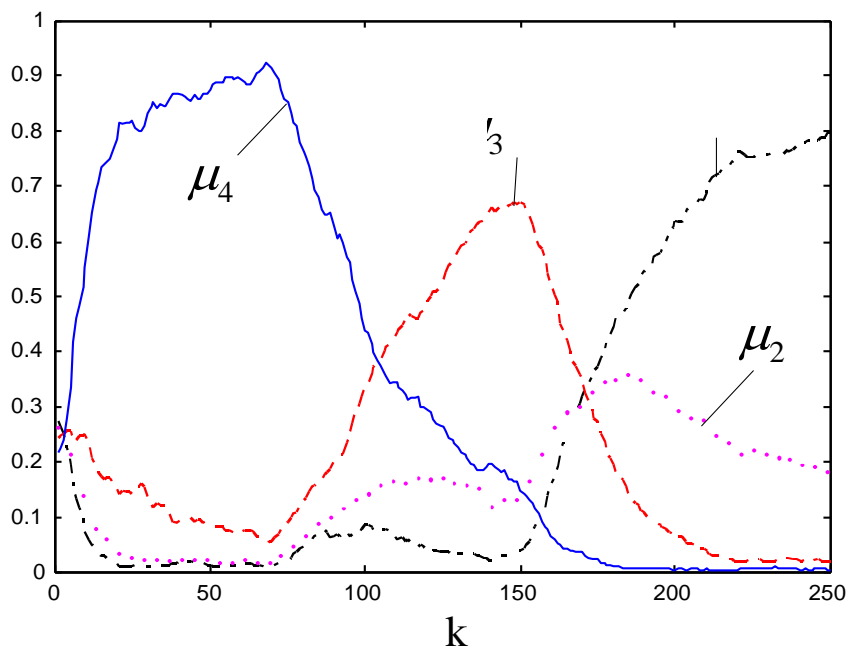

Figure 6: Average IMM mode probabilities 


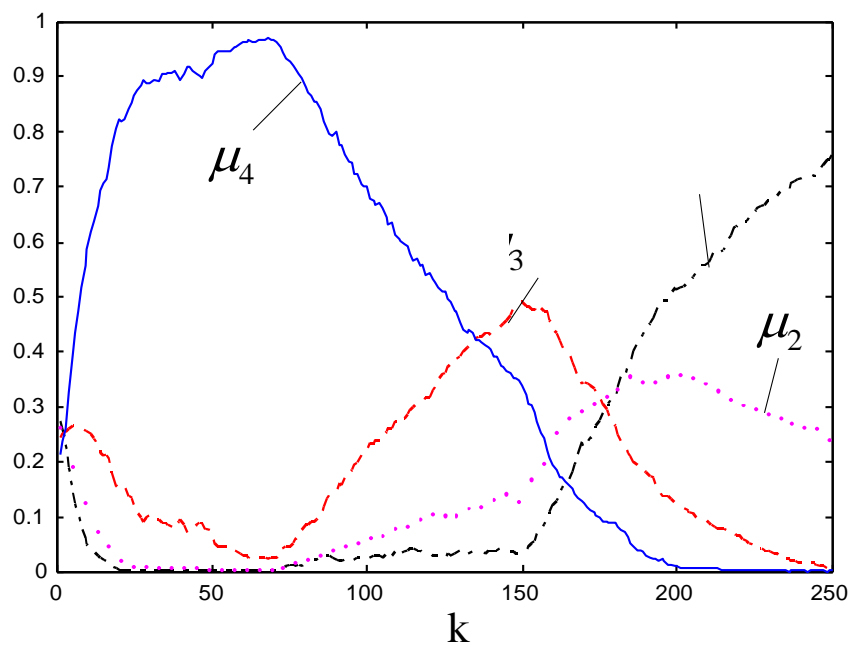

Figure 7: Average MMAC mode probabilities

In both simulation experiments the cost function of the IMM algorithm for control is smaller than the respective MMAC values. The obvious priority of the IMM algorithm for control is due to the faster response to abrupt changes in the parameters (see Fig.6 and Fig.7). On the basis of the simulation experiments it can be concluded that in stationary conditions the results of both algorithms are comparable, but in the nonstationary case, the IMM algorithm for control yields better overall performance than the MMAC algorithm with respect to fast response and reliability.

\section{Conclusions}

An Interacting Multiple Model (IMM) algorithm for stochastic systems control in the presence of parametric model uncertainty is designed, for stationary and nonstationary systems. It is based on the cost-effective IMM estimator. The overall system control is synthesized as a probabilistically weighted sum of the control processes received from separate regulators. These regulators are synthesised for each model from the uncertainty domain. The overall control process is computed as a state feedback. The well known and cost effective IMM filter is used for partial state estimates generation. The IMM partial state estimates are used by regulators working in parallel to compute the partial control processes and the common state feedback. Each regulator is synthesised based on a quadratic cost function minimization. Results from simulation experiments are given. The algorithm presented is compared to other MM algorithm for control of Bayesian type. The simulation results demonstrate that the IMM algorithm for control provides better results in the 
presence of abrupt changes in the parameters than the MMAC algorithm. The performance of both algorithms is comparable in a stationary mode.

Acknowledgement: The work on this paper was partially supported by contract No.I808/98 with the Bulgarian Science Fund.

1. Athans, M., D. Castañon, et al., "The Stochastic Control of the F-8C Aircraft Using a MMAC Method - I: Equilibrium Flight," IEEE Transaction on Automatic Control 22, 5 (1977), 768- 780.

2. Bar-Shalom, Y. and Xiao Rong-Li, Multitarget-Multisensor Tracking: Principles and Techniques (Storrs, CT: YBS Publishing, 1995).

3. Griffin, G., Jr. and P. Maybeck, "MMAE/ MMAC Control for Bending with Multiple Uncertainty Parameters,” IEEE Trans. AES 33, 3 (1997), 903-912.

4. Gustafson, J. and P. Maybeck, "Flexible Space Structure Control Via Moving-Bank Multiple Model Algorithms," IEEE Trans. AES 30, 3 (1994), 750-757.

5. Xiao Rong-Li, "Hybrid Estimation Techniques," in Control and Dynamic Systems, vol.76, ed. C.T. Leondes (Academic Press, Inc., 1996), 213-287.

6. Madjarov, N. and L. Mihaylova, "Adaptive Multiple Model Algorithms for Estimation and Control of Stochastic Systems," Automatics \& Informatics, 1/2 (1993), 20-23 (in Bulgarian).

7. Maybeck, P. and K. Hentz, "Investigation of Moving-Bank Multiple Model Adaptive Algorithms," AIAA J. Guidance, Control \& Dynamics 10 (1987), 90-96.

8. Maybeck, P. and R. Stevens, "Reconfigurable Flight Control Via Multiple Model Adaptive Control Methods," IEEE Trans. AES 27 (May 1991), 470-480.

9. Mazor, E., A. Averbuch, Y. Bar-Shalom and J. Dayan, "IMM Methods in Target Tracking: A Survey," IEEE Trans. AES 34, 1 (1998), 103-123.

10. Murray-Smith, R. and T.A. Johansen, eds., Multiple Model Approaches to Modelling and Control (Taylor \& Francis, 1997).

11. Narendra, K. and J. Balakrishnan, "Adaptive Control Using Multiple Models," IEEE Transactions on Automatic Control 42, 2 (1997), 171-187.

12. Schiller, G. and P. Maybeck, "Control of a Large Space Structure Using MMAE/ MMAC Techniques," IEEE Trans. on AES 33, 4 (1997), 1122-1130.

13. Zhang, Y. and Xiao Rong-Li, "Detection and Diagnosis of Sensor and Actuator Failures Using IMM Estimator,” IEEE Trans. on AES 34, 4 (1998), 1293-1311.

14. Xiao, Q., M. Rao, Y. Ying and S. Shen, "Adaptive Fading Kalman Filter with an Application," Automatica 30, 8 (1994), 1333-1338. 